\title{
Biomechanical Problems Related to the Pedicle Screw System
}

\author{
Tetsutaro MIZUNO'1, Toshihiko SAKAKIBARA¹, Takamasa YOSHIKAWA², Tadashi INABA², Takaya KATO², \\ Yuichi KASAI ${ }^{1}$
}

${ }^{1}$ Mie University Graduate School of Medicine, Department of Spinal Surgery and Medical Engineering, Japan

${ }^{2}$ Mie University, Graduate School of Engineering, Department of Mechanical Engineering, Japan

${ }^{3}$ Mie University, Community-University Research Cooperation Center, Japan

\section{ABSTRACT}

AIM: To assess biomechanical problems related to pedicle screw (PS) systems.

MATERIAL and METHODS: Functional spinal units (L3-4) of deer were evaluated using a 6-axis material testing machine. For the specimen models, we prepared an intact model, a damaged model, a PS model, and a crosslink model. We checked the range of motion (ROM) during bending and rotation tests. Eight directions were measured in the bending test: anterior, right-anterior, right, right-posterior, posterior, left-posterior, left, and left-anterior, and 2 directions were measured in the rotation test (right and left).

RESULTS: ROMs of the PS model were smaller than those of the intact model in all directions. However, ROMs of the PS model in the rotation test were smaller than those of the damaged model and larger than those of the intact model. The stability of the crosslink model was better than that of the PS model during the bending test, but ROMs of the crosslink model were larger than those of the intact model during the rotation test.

CONCLUSION: Excessive bending rigidity and rotational instability are the biomechanical problems related to PS systems. Based on these results, we speculate that one of the most significant causes of adjacent segment disease is excessive bending rigidity and one of the most important causes of instrumentation failure is rotational instability.

KEYWORDS: Biomechanics, Bending rigidity, Lumbar spine, Pedicle screw, Rotational instability

ABBREVIATIONS: PS: Pedicle screw, ROM: Range of motion, ASD: Adjacent segment disease

\section{INTRODUCTION}

S pinal instability is typically the result of degenerative changes, injuries, or surgical procedures. Pedicle screw (PS) systems are the most widely used products for instrumentation in the treatment of spinal instability; however, complications of these systems including adjacent segment disease and instrumentation failure are often reported and might be caused by biomechanical problems. We developed a 6-axis material testing machine and performed various experiments to examine spinal biomechanics (2).
We typically compare a damaged model with a fixation model of the spine. However, assuming that the ideal objective of spinal instrumentation is to stabilize the damaged spine in a manner closer to that of an intact spine, it is important to compare the fixation model with a model of an intact spine. For example, the utility of the crosslink system for torsional spine stability is widely known, but few studies have compared a model of intact spine with a model of spine fixed with a PS or crosslink system. We found no studies considering this point of view. Consequently, we investigated the biomechanical problems related to PS systems by comparing a PS fixation model with that of the intact spine. 


\section{MATERIAL and METHODS}

This study was performed with the approval (No. 1544) of the Research Ethics Committee in Mie University Graduate School of Medicine.

We used functional spinal units (L3-4) of deer as specimens. The deer were culled as part of a wildlife management program. The lumbar vertebrae were initially cryogenically preserved at $-30^{\circ} \mathrm{C}$. The muscles and fat were removed while the internal stabilizing elements were retained after thawing each of the frozen lumbar spine at room temperature. The cranial and caudal portions of each specimen were fixed to a jig with dental resin. For the specimen models, we prepared an intact model, a damaged model, a PS model, and a crosslink model. Internal stabilizing elements were retained in the intact model. The damaged model was prepared by drilling holes (3-mm in diameter) at sites one-fourth, one-half, and three-fourths of the distance from the anterior surface of the intervertebral disc and removing the supraspinous ligament, interspinous ligament, and both facet joints. The PS model was similar to the damaged model but was fixed with $\phi 5.5 \times 35 \mathrm{~mm}$ pedicle screws and rods (5- $\mathrm{mm}$ in diameter) (KISCO Co Ltd., SUIREN ${ }^{\circledR}$, Kobe, Japan). The crosslink model was fixed using both a PS system and a crosslink system (Medtronic, TSRH ${ }^{\circledR} \mathrm{RP}$, Memphis, TN, USA) (Figure 1). We tested 16 specimens to compare the intact, damaged, and PS models in Study 1 and 13 specimens to compare the intact, model, and crosslink models in Study 2.
We used the 6-axis material testing machine developed in our laboratory, which utilizes a parallel mechanism for biomechanical testing (Figure 2). Three sets of 2 actuators each are located parallel to each other and another set is equidistantly disposed; each of the 6 actuators is independently controlled. A 6-axis force sensor is placed at the site where the cranial jig is connected to detect forces in the $\mathrm{x}, \mathrm{y}$, and $z$ axes as well as the torque around each axis. Furthermore, this force sensor enables force/torque control via feedback of the detected results into the control system. In this study, we performed both a bending test (anterior, right-anterior, right, right-posterior, posterior, left-posterior, left, and left-anterior bending) and a rotation test (right and left rotation), and each test was repeated twice. Torque was set at -3.0 to $3.0 \mathrm{Nm}$ for the bending test and -5.0 to $5.0 \mathrm{Nm}$ for the rotation test and was loaded at an angular speed of 0.1 degrees/s. Superior vertebral body displacement/angular displacement and generated force/torque were determined for each test and recorded in the computer (sample cycle, $1 \mathrm{~Hz}$ ). We defined superior vertebral angular displacement as the result recorded when maximum torque was loaded as the range of motion (ROM) and compared the ROM of each model.

We used the Friedman test to investigate intergroup differences among the 3 models in each study. A post hoc analysis was performed using the Steel-Dwass test for multiple comparisons among the 3 groups when the overall differences were statistically significant, and the level of statistical significance was set at $\mathrm{p}<0.05$.

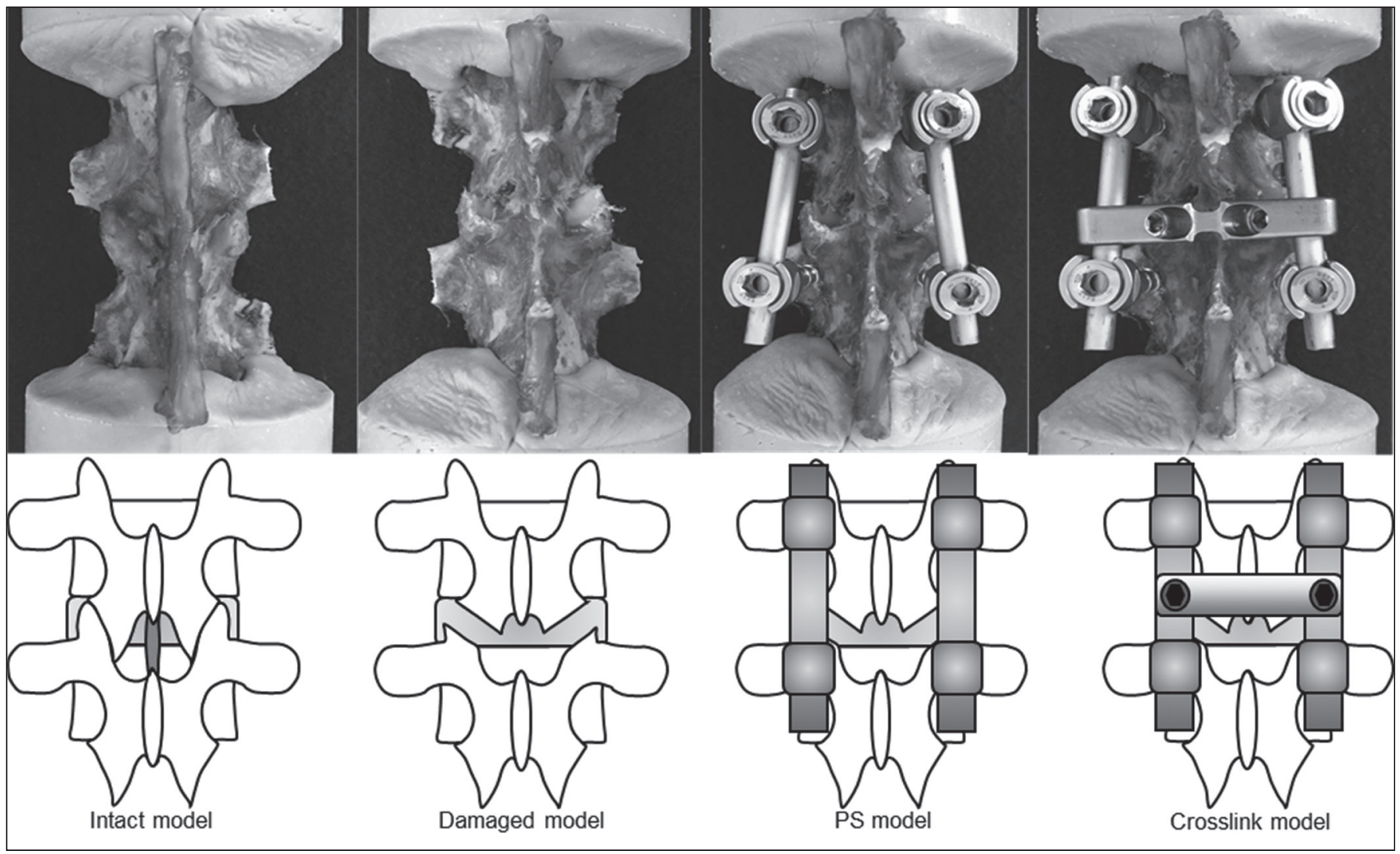

Figure 1: Schemas and pictures of each model. 


\section{RESULTS}

The bending test results and the average ROM of each model are illustrated as radar charts in Figures 3 and 4. The rotation test results and the average ROM of each model with the standard deviation are shown as bar charts in Figures 5 and 6. Tables I and II show the results of multiple comparisons.

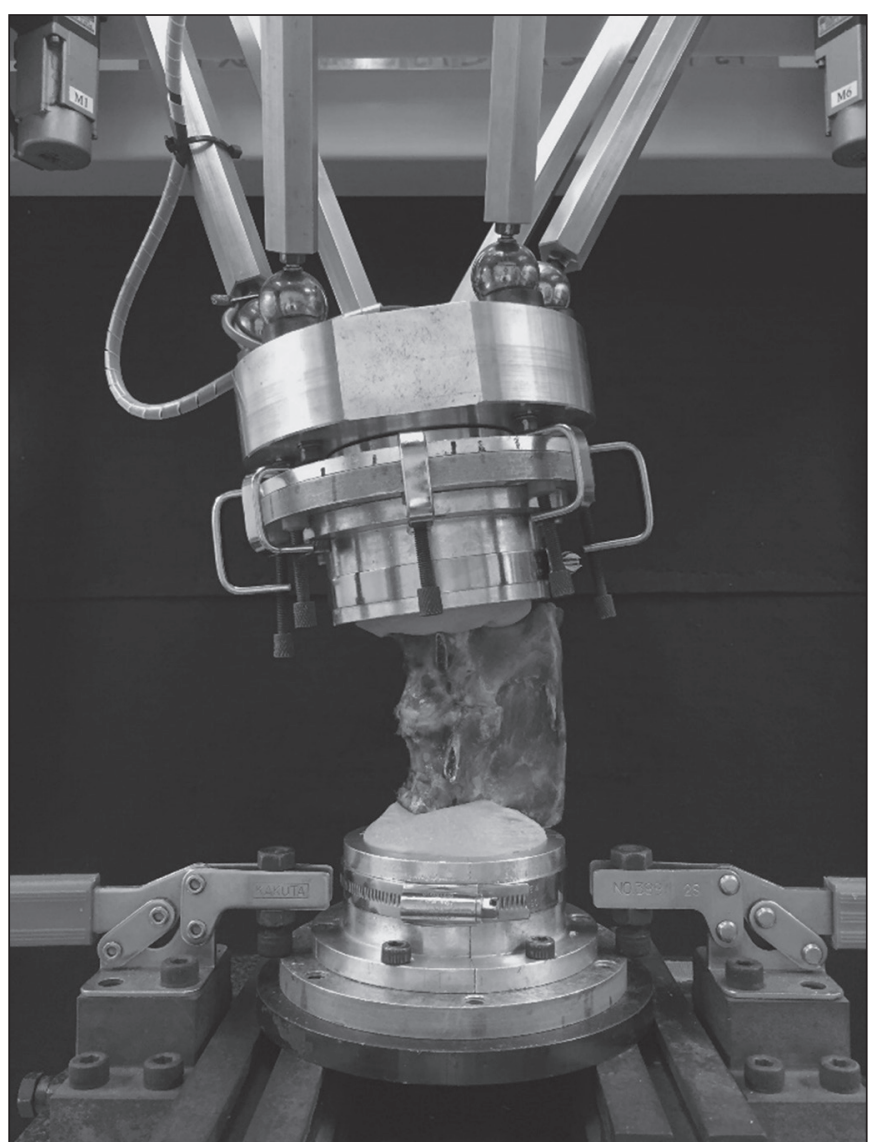

Figure 2: A 6-axis material testing machine during flexion test with the intact model.

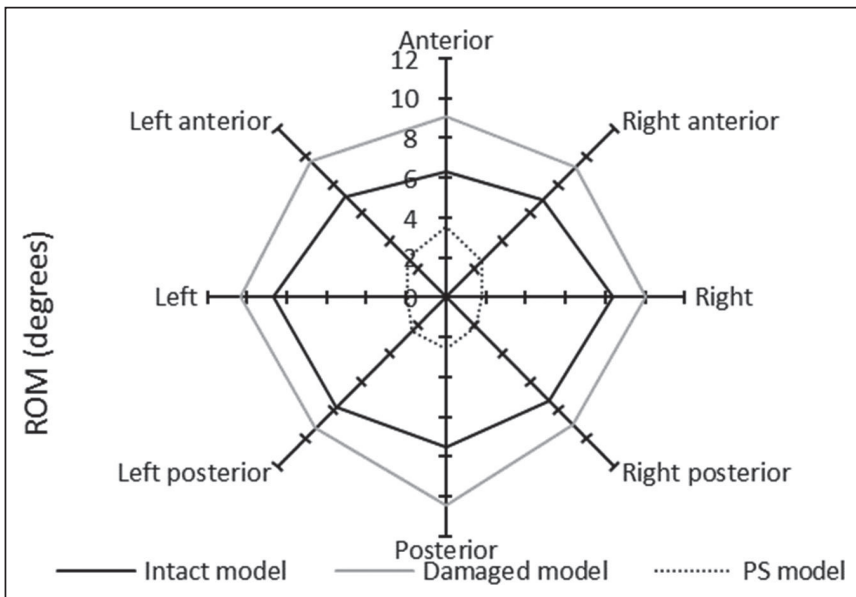

Figure 3: The chart shows average ROMs of each model during the bending test for Study 1.
Figures 3 and 5 as well as Table I show the results of Study 1 , and figures 4 and 6 as well as Table II show the results of Study 2.

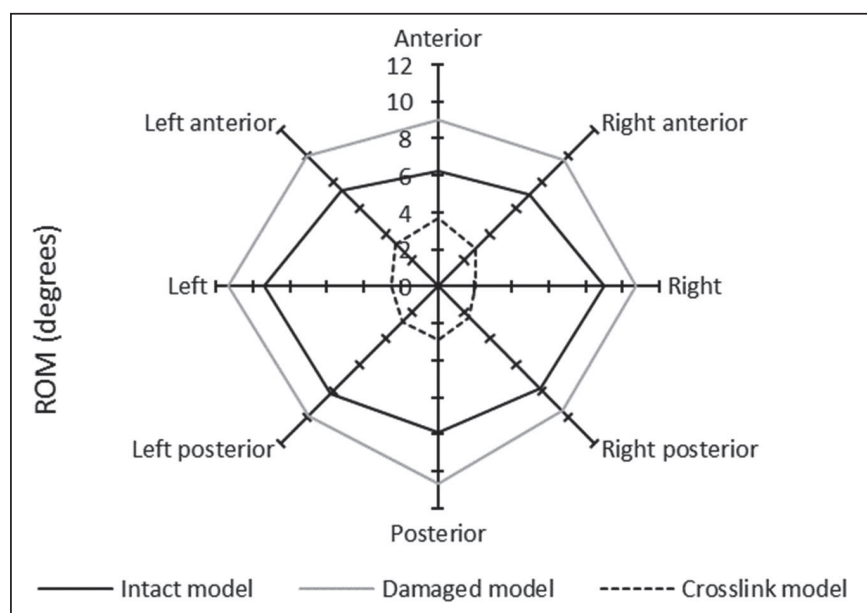

Figure 4: The chart shows average ROMs of each model during the bending test for Study 2 .

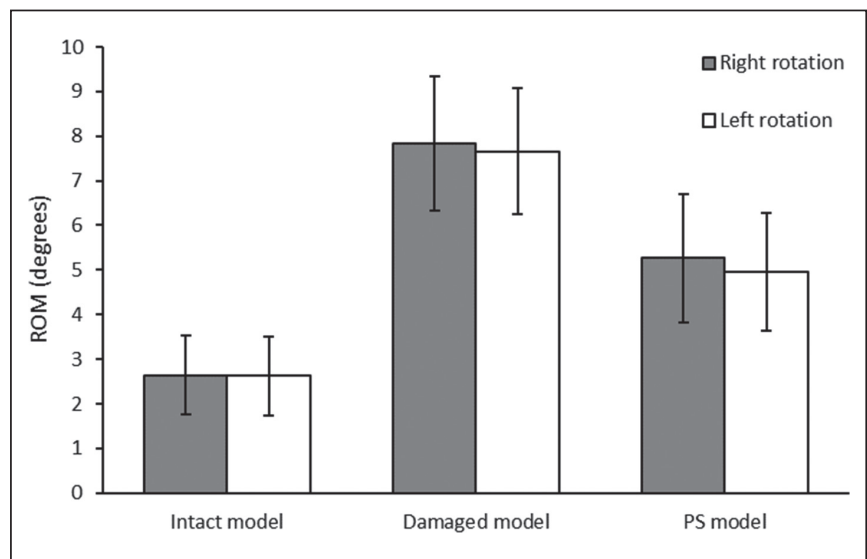

Figure 5: The chart shows average ROMs and standard deviations of each model during the rotation test for Study 1 .

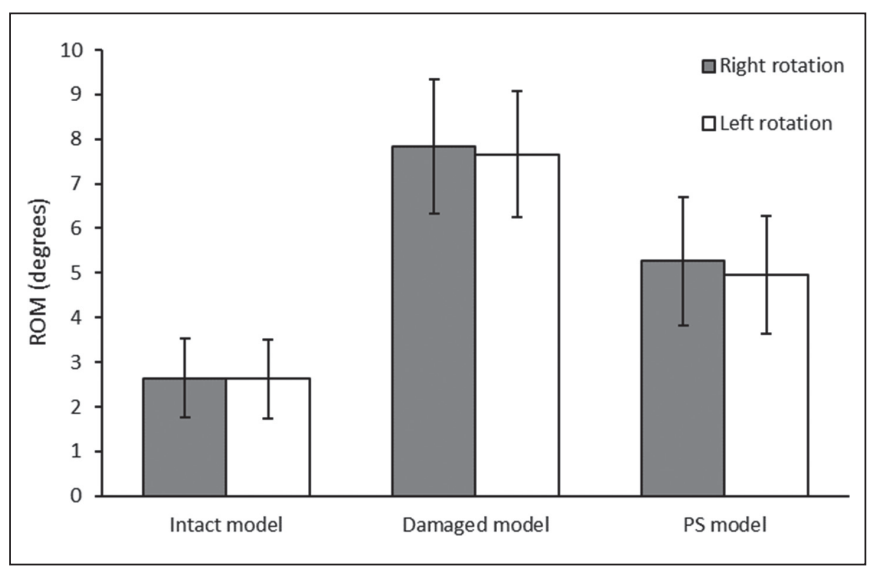

Figure 6: The chart shows average ROMs and standard deviations of each model during the rotation test for Study 2 . 
Table I: The Results of Multiple Comparisons Between Intact Model, Damaged Model and PS Model

\begin{tabular}{|c|c|c|c|c|c|c|c|c|c|c|}
\hline & Anterior & $\begin{array}{c}\text { Right } \\
\text { anterior }\end{array}$ & Right & $\begin{array}{c}\text { Right } \\
\text { posterior }\end{array}$ & Posterior & $\begin{array}{c}\text { Left } \\
\text { posterior }\end{array}$ & Left & $\begin{array}{c}\text { Left } \\
\text { anterior }\end{array}$ & $\begin{array}{c}\text { Right } \\
\text { rotation }\end{array}$ & $\begin{array}{c}\text { Left } \\
\text { rotation }\end{array}$ \\
\hline Intact - Damaged & * * & * * & & * * & * * & * & & * * & * * & * * \\
\hline PS - Intact & * * & ** & ** & * * & * * & * * & * * & * * & * * & * * \\
\hline
\end{tabular}

* Statistical significance (Level of significance $<5 \%$ ).

* * Statistical significance (Level of significance $<1 \%$ ).

Table II: The Results of Multiple Comparisons Between Intact Model, Damaged Model and Crosslink Model

\begin{tabular}{|c|c|c|c|c|c|c|c|c|c|c|}
\hline & Anterior & $\begin{array}{c}\text { Right } \\
\text { anterior }\end{array}$ & Right & $\begin{array}{c}\text { Right } \\
\text { posterior }\end{array}$ & Posterior & $\begin{array}{c}\text { Left } \\
\text { posterior }\end{array}$ & Left & $\begin{array}{c}\text { Left } \\
\text { anterior }\end{array}$ & $\begin{array}{l}\text { Right } \\
\text { rotation }\end{array}$ & $\begin{array}{c}\text { Left } \\
\text { rotation }\end{array}$ \\
\hline Intact - Damaged & * * & * * & * & * * & * * & * * & * & * * & * * & * * \\
\hline Damaged - Crosslink & * * & * * & * * & * * & * * & * * & * * & * * & * * & * * \\
\hline Crosslink - Intact & * * & * * & * * & * * & * * & * * & * * & ** & * * & * * \\
\hline
\end{tabular}

* Statistical significance (Level of significance $<5 \%$ ).

* * Statistical significance (Level of significance $<1 \%$ ).

\section{Study 1}

For the bending test, the average ROMs of the intact and damaged PS model were $6.3^{\circ}, 9.0^{\circ}$, and $3.5^{\circ}$ during anterior bending; $6.9^{\circ}, 9.2^{\circ}$, and $2.6^{\circ}$ during right anterior bending; $8.4^{\circ}, 10^{\circ}$, and $1.8^{\circ}$ during right bending; $7.3^{\circ}, 9.0^{\circ}$, and $2.1^{\circ}$ during right posterior bending; $7.5^{\circ}, 10^{\circ}$, and $2.6^{\circ}$ during posterior bending; $7.8^{\circ}, 9.3^{\circ}$, and $2.4^{\circ}$ during left posterior bending; $8.7^{\circ}, 10^{\circ}$, and $2.0^{\circ}$ during left bending; and $7.2^{\circ}$, $9.7^{\circ}$, and $2.8^{\circ}$ during left anterior bending, respectively.

For the rotation test, the average ROMs of the intact and damaged PS model were $2.6^{\circ}, 7.8^{\circ}$, and $5.3^{\circ}$ during right rotation and $2.6^{\circ}, 7.7^{\circ}$, and $5.0^{\circ}$ during left rotation, respectively.

The ROMs for the damaged model were larger than for the intact model in all directions during the bending and rotation tests. The ROMs for the PS model were smaller than for the damaged model in all directions during the bending and rotation tests; they were also smaller than for the intact model in all directions during the bending test but larger than those for the intact model during the rotation test. The Friedman and Steel-Dwass tests showed significant differences in all comparisons except when comparing the intact model with the damaged model in the right and left bending test.

\section{Study 2}

For the bending test, the average ROMs of the intact, damaged, and crosslink model were $6.2^{\circ}, 9.0^{\circ}$, and $3.7^{\circ}$ during anterior bending; $7.0^{\circ}, 9.7^{\circ}$, and $2.9^{\circ}$ during right anterior bending; $9.0^{\circ}, 11^{\circ}$, and $2.0^{\circ}$ during right bending; $7.8^{\circ}, 9.5^{\circ}$, and $2.4^{\circ}$ during right posterior bending; $7.9^{\circ}, 11^{\circ}$, and $2.9^{\circ}$ during posterior bending; $8.2^{\circ}, 9.9^{\circ}$, and $2.7^{\circ}$ during left posterior bending; $9.4^{\circ}, 11^{\circ}$, and $2.5^{\circ}$ during left bending; and $7.4^{\circ}, 10^{\circ}$, and $3.2^{\circ}$ during left anterior bending, respectively.
For the rotation test, the average $\mathrm{ROMs}$ of the intact, damaged, and crosslink model were $3.0^{\circ}, 8.1^{\circ}$, and $4.2^{\circ}$ during right rotation and $3.0^{\circ}, 8.3^{\circ}$, and $4.3^{\circ}$ during left rotation, respectively.

The ROMs for the damaged model were larger than for the intact model in all directions during the bending and rotation tests, those for the crosslink model were smaller than for the damaged model in all directions during the bending and rotation tests, and those for the crosslink model were also smaller than for the intact model in all directions during the bending test, but larger than for the intact model during the rotation test. The results of the Friedman and Steel-Dwass tests were significantly different in all comparisons.

\section{DISCUSSION}

PS systems have been widely used and the number of surgeries has increased in recent years due to advances in implant technology; however, the rate of adjacent segment disease (ASD) and instrumentation failure is approximately $10 \%-20 \%$, which is a problem that cannot be overlooked $(5,14,15)$.

In this study, ROMs of the PS model during the bending test were less than half of those of the intact model. It appears that a PS system provides good stability of the damaged spine. Conversely, ROMs of the PS model were approximately twice as large as those of the intact model in the rotation test. ROMs of the crosslink model were also larger than those of the intact model in the rotation test and the differences were statistically significant. Based on the findings of the rotation test, we determined that the stability attained with a PS or crosslink system was insufficient. Considering that one of the main objectives of spinal instrumentation is to restore normal movement, we concluded that the rigidity observed in the 
PS and crosslink models in the bending test was excessive whereas it was insufficient in the rotation test. When we considered the biomechanical characteristics of the PS system, we speculated that excessive bending rigidity was one of the most significant causes of ASD and rotational instability was one of the most important causes of instrumentation failure; we found few studies considering this point of view.

When considering excessive bending stability, Park et al. reported that $12 \%$ to $18 \%$ of patients who had undergone PS instrumentation developed ASD and Cheh et al. reported a rate of $30.3 \%(1,14)$. We previously performed an experiment with boar lumbar spine (L3-4) and reported that triple torque was needed to bend the spine in the PS model in comparison with the intact model (12), suggesting that the excessive stability seen in the bending test might be a cause of ASD.

Graf ligamentoplasty and Dynesys ${ }^{\circledR}$ Dynamic Stabilization System were developed as solutions for excessive bending rigidity. Kitahara et al. conducted a biomechanical experiment and reported that movement of adjacent segments with Graf ligamentoplasty was smaller than with PS instrumentation (7). Kanayama et al. and Hadlow et al. compared clinical results between Graf ligamentoplasty and PS instrumentation and reported that there were no significant differences between the 2 procedures $(4,6)$. However, in these reports, the surgeons performed these procedures in patients with different surgical indications, and Graf ligamentoplasty had some unfavorable results even though there were no significant differences. Thus, based on the clinical outcome, Graf ligamentoplasty might not be considered an effective procedure. Niosi et al. performed a biomechanical experiment testing the Dynesys ${ }^{\circledR}$ Dynamic Stabilization System and reported that it almost normalized movement in the injury model in both bending and rotation motions (13). However, Grob et al. reported that the rates of improvement in the ability to engage in physical activities and in quality of daily life were no more than $50 \%$ and $19 \%$, respectively, in patients who required or were scheduled for further surgical intervention within a 2-year follow-up (3).

When considering instability in the rotation test, Thomsen et al. reported that $21 \%$ of patients who underwent lumbar monosegmental fixation with a PS system did not achieve fusion, and Jutte et al. reported that $12 \%$ of patients who underwent lumbosacral fusion suffered screw breakages $(5,15)$, suggesting that rotational instability was a cause of instrumentation failure.

We typically use a crosslink system as a solution for rotational instability and many biomechanics studies support this application $(11,16)$. Wang et al. reported that 1 crosslink might restrict rotation and 2 crosslinks may provide better fixation regardless of the position of the second crosslink (18). Wahba et al. compared an intact model with a crosslink model using human cadaver corpectomy models and reported that crosslinking provided improved stability but did not reach the level of stability of the intact model (17). We did not find an experiment comparing an intact model with a PS model with 2 crosslinks; however, even the use of 2 crosslinks might not bring stability close to that of an intact model.
As yet, there are no suitable solutions for excessive bending rigidity and rotational instability related to a PS system. The development of PS systems, which provide strong stability by passing screws through the pedicle, has led to advances in spinal instrumentation surgery; however, these systems are also generating new problems.

The limitations of this study are that the damaged model may have had too much instability secondary to removal of both facet joints and that we used cadaveric deer spines as specimens. In recent years, it has become more difficult to use human cadavers for experiments in Japan, so we have resorted to using deer and boars instead. Since it cannot be said that spinal autopsy is similar between deer and humans, it is impossible to compare the biomechanical data simply by ROM. However, Wasinpongwanich et al. found when examining the ROM change rate, an index evaluating how intervertebral stability will change when normal spine of deer is injured or fixed by instrumentation, in normal, damaged, and PS fixation models in deer that it does approximate that of humans (19). In experiments exploring the biomechanical tendency, as in our study, the spine of culled deer is therefore considered an alternative available to humans (8-10).

\section{- CONCLUSION}

Biomechanical complications of PS systems include excessive bending rigidity and rotational instability. We speculated that one of the most significant causes of ASD was excessive bending rigidity and one of the most important causes of instrumentation failure was rotational instability. We propose that ideal spinal instrumentation should be less rigid during bending and more rigid during rotation than the PS system; these refinements could lead to superior spinal instrumentation in the future.

\section{- REFERENCES}

1. Cheh G, Bridwell KH, Lenke LG, Buchowsli JM: Adjacent segment disease following lumbar/thoracolumbar fusion with pedicle screw instrumentation. Spine 32(20): 2253-2257, 2007

2. Fujiwara M, Masuda T, Inaba T, Katoh T, Kasai $Y$, Satoru I: Development of 6-axis material tester for measuring mechanical spine properties. Journal of Robotics and Mechatronics 18(2):160-165, 2006

3. Grob D, Benini A, Junge A, Mannion AF: Clinical experience with the Dynesys semirigid fixation system for the lumbar spine. Spine 30(3):324-331, 2005

4. Hadlow SV, Fagan AB, Hillier TM, Fraser RD: The Graf ligamentoplasty procedure. Spine 23(10):1172-1179, 1998

5. Jutte PC, Castelein RM: Complication of pedicle screws in lumbar and lumbosacral fusions in 105 consecutive primary operations. Eur Spine J 11:594-598, 2002

6. Kanayama M, Hashimoto $T$, Shigenobu $K$, Harada $M$, Oha F, Ohkoshi Y, Tada H, Yamamoto K, Yamane S: Adjacentsegment morbidity after Graf ligamentoplasty compared with posterolateral lumbar fusion. J Neurosurg 95:5-10, 2001 
7. Kitahara K, Uni K, Hasegawa K, Takano K, Hara T: Biomechanical effect of fusion and stabilization on the adjacent segments in the lumbar spine. Journal of Japanese Society for Clinical Biomechanics 25:133-137, 2004

8. Kumar N, Kukreti S, Ishaque M, Mulholland R: Anatomy of deer spine and its comparison to the human spine. Anat Rec 260:189-203, 2000

9. Kumar N, Kukreti S, Ishaque M, Sengupta DK, Mulholland R: Functional anatomy of the deer spine: An appropriate biomechanical model for the human spine? Anat Rec 266:108117, 2002

10. Liu GM, Li YQ, Xu CJ, Zhu XM, Liu Y: Feasibility of vertebral internal fixation using deer and sheep as animal models. Chin Med J (Engl) 123(17):2379-2383, 2010

11. Lynn G, Mukherjee DP, Kruse RN, Sadasivan KK, Albright JA: Mechanical stability of thoracolumbar pedicle screw fixation. The effect of crosslink. Spine 22(14):1568-1573, 1997

12. Masaoka $T$, Inaba $T$, Kasai $Y$, Uchida $A$, Mogi $M$, Kato $T$, Nakamata T, Tokuda M: Biomechanical effect of spinal instrumentation on adjacent segments. Journal of Japanese Society for Clinical Biomechanics 29:305-310, 2008

13. Niosi CA, Zhu QA, Wilson DC, Keynan O, Wilson DR, Oxland TR: Biomechanical characterization of the three-dimensional kinematic behavior of the Dynesys dynamic stabilization system: An in vitro study. Eur Spine J 15:913-922, 2006
14. Park P, Garton HJ, Gala VC, Hoff JT, McGillicuddy JE: Adjacent segment disease after lumbar or lumbosacral fusion: Review of the literature. Spine 29(17):1938-1944, 2004

15. Thomsen K, Christensen FB, Eiskjer SP, Hansen ES, Fruensgaard S, Bunger CE: The effect of pedicle screw instrumentation on functional outcome and fusion rates in posterolateral lumbar spinal fusion: A prospective, randomized clinical study. Spine 22(24):2813-2822, 1997

16. Valdevit A, Kambic HE, McLain RF: Torsional stability of crosslink configurations: A biomechanical analysis. Spine J 5:441445, 2005

17. Wahba GM, Bhatia N, Bui CN, Lee KH, Lee TQ: Biomechanical evaluation of short-segment posterior instrumentation with and without crosslinks in a human cadaveric unstable thoracolumbar burst fracture model. Spine 35(3):278-285, 2010

18. Wang Z, Sakakibara T, Yoshikawa T, Inaba T, Kasai Y: Do the position and orientation of the crosslink influence the stiffness of spinal instrumentation? Clin Spine Surg 30(4):176-180, 2017

19. Wasinpongwanich K, Sakakibara T, Yoshikawa T, Inaba T, Kasai Y: Are deer and boar spines a valid biomechanical model for human spines? J Spine 3:187, 2014 